Advances in Dynamical Systems and Applications (ADSA).

ISSN 0973-5321, Volume 15, Number 1, (2020) pp. 29-33

(C) Research India Publications

https://dx.doi.org/10.37622/ADSA/15.1.2020.29-33

\title{
A Proof with Respect to Laplace Transform of the $n$-th Derivative by Mathematical Induction
}

\author{
Hwajoon Kim \\ Kyungdong University, 11458, Kyungdong Univ. Rd. 27, Yangju, Gyeonggi, S. Korea.
}

\begin{abstract}
In this study, we consider a proof with respect to the Laplace transform of the $n$-th derivative of any order by mathematical induction. The content with respect to the Laplace transform of the $n$-th derivative is widely used without a strict proof. Hence, we propose a proof by applying the rigor of mathematical induction.
\end{abstract}

AMS Subject Classification: 44A05, 35A22

Keywords and Phrases: generalized integral transform, transform of derivative, induction.

\section{INTRODUCTION}

The Laplace transform method is a popular tool for solving linear ordinary differential equations and finding analytical solutions of partial differential equations. Although the Laplace transform method is fairly logical, there are some weaknesses in terms of the integrability and existence as several hypotheses are required when using the method. Therefore, this area should be further investigated. Moreover, the proof with respect to the Laplace transform of the derivatives is not strict. Hence, in this study, we propose a proof with respect to the Laplace transform of the $n$-th derivative by induction, and the proposed proof is strict unlike the existing proofs. Additionally, this result can be extended to a generalized integral transform or other transforms. This study is fairly simple, and was conducted because of an academic interest. 
There are several Laplace-type integral transforms, and a generalized integral transform $[4,11]$ can be expressed as follows;

$$
G £(f)=u^{\alpha} \int_{0}^{\infty} e^{-\frac{t}{u}} f(t) d t
$$

where $u=G(f)$. Most existing Laplace-type integral transforms[2, 10, 12] are interpreted as variants of the Laplace transform, whereas the Mellin transform[1] can be considered relatively reasonable. From a review of related studies, Jhanthanam et al. studied the generalized solutions of a third-order Cauchy-Euler equation using the Laplace transform[3], determined an application of the above generalized Laplace transform in partial differential equations[7], and obtained the solution of a heat equation without boundary conditions[6]. The solution of Laguerre's equation was studied using the Laplace-type transform[5], and generalized hypergeometric functions have been obtained by considering a new class of the above Laplace-type integrals[8].

The following results are obtained using the induction.

(1) (Laplace transform of the $n$-th derivative of any order) Let $f, f^{\prime}, \cdots, f^{(n-1)}$ be continuous for all $t \geq 0$ and satisfy the growth restriction[9]. Furthermore, let $f^{(n)}$ be piecewise continuous on every finite interval on the semi-axis $t \geq 0$. Then, the transform of $f^{(n)}$ satisfies the following equation;

$$
£\left(f^{(n)}\right)=s^{n} £(f)-\sum_{k=1}^{n} s^{n-k} f^{(k-1)}(0),
$$

where $f^{(n)}$ is the $n$-th derivative of $f$.

(2) This theorem can be extended to the above generalized Laplace transform as follows;

$$
\begin{gathered}
G_{\alpha}\left(f^{(n)}\right)=\frac{F(x, u)}{u^{n}}-u^{\alpha}\left[\frac{1}{u^{n-1}} f(x, 0)+\frac{1}{u^{n-2}} \frac{\partial}{\partial t} f(x, 0)+\cdots\right. \\
\left.+\frac{1}{u} \frac{\partial^{n-2}}{\partial t^{n-2}} f(x, 0)+\frac{\partial^{n-1}}{\partial t^{n-1}} f(x, 0)\right],
\end{gathered}
$$

where $F(x, u)=G(x, t)$ and $\partial^{0} / \partial=1$.

\section{A PROOF WITH RESPECT TO LAPLACE TRANSFORM OF THE $n$-th DERIVATIVE BY MATHEMATICAL INDUCTION}

We consider a proof with respect to Laplace transform of the $n$-th derivative of any order by mathematical induction. 
Lemma 1. (growth restriction[9]) A function $f(t)$ satisfies the growth restriction if

$$
|f(t)| \leq M e^{k t}
$$

for all $t \geq 0$ and some constants $M$ and $k$.

Theorem 2. (Laplace transform of the $n$-th derivative of any order) Let $f, f^{\prime}, \cdots$, $f^{(n-1)}$ be continuous for all $t \geq 0$ and satisfy the growth restriction[10]. Furthermore, let $f^{(n)}$ be piecewise continuous on every finite interval on the semi-axis $t \geq 0$. Then the transform of $f^{(n)}$ satisfies

$$
£\left(f^{(n)}\right)=s^{n} £(f)-s^{n-1} f(0)-s^{n-2} f^{\prime}(0)-\cdots-f^{(n-1)}(0),
$$

where $f^{(n)}$ is the $n$-th derivative of $f$.

Proof. Let us represent the equation $(*)$ as a contracted form;

$$
£\left(f^{(n)}\right)=s^{n} £(f)-\sum_{k=1}^{n} s^{n-k} f^{(k-1)}(0),
$$

where $f^{(n)}$ is the $n$-th derivative of $f$. Next, let us approach the proof by induction. In case of $n=1$,

$$
£\left(f^{\prime}\right)=\int_{0}^{\infty} e^{-s t} f^{\prime}(t) d t=s £(f)-f(0)
$$

and this is the same as the value of $\left(^{*}\right)$. Next, let us suppose that $n=m$ is valid for some $m$. Thus,

$$
£\left(f^{(m)}\right)=s^{m} £(f)-\sum_{k=1}^{m} s^{m-k} f^{(k-1)}(0)
$$

holds for $f^{(m)}$ is the $m$-th derivative of $f$. Let us show that

$$
£\left(f^{(m+1)}\right)=s^{m+1} £(f)-\sum_{k=1}^{m+1} s^{m+1-k} f^{(k-1)}(0) .
$$

Differentiating (**), we have

$$
\begin{gathered}
£\left(f^{(m+1)}\right)=s £\left(f^{(m)}\right)-f^{(m)}(0) \\
=s\left[s^{m} £(f)-\sum_{k=1}^{m} s^{m-k} f^{(k-1)}(0)\right]-f^{(m)}(0) \\
=s^{m+1} £(f)-\sum_{k=1}^{m} s^{m+1-k} f^{(k-1)}(0)-f^{(m)}(0) \\
=s^{m+1} £(f)-\sum_{k=1}^{m+1} s^{m+1-k} f^{(k-1)}(0) .
\end{gathered}
$$

Hence, this theorem is valid at an arbitrary natural number $n$. 
This theorem can be extended to the above generalized transform as follows;

$$
\begin{gathered}
G_{\alpha}\left(f^{(n)}\right)=\frac{G_{\alpha}(f)}{u^{n}}-u^{\alpha}\left[\frac{1}{u^{n-1}} f(0)+\frac{1}{u^{n-2}} f^{\prime}(0)+\cdots\right. \\
\left.+\frac{1}{u} f^{(n-2)}(0)+f^{(n-1)}(0)\right],
\end{gathered}
$$

where $f^{(n)}$ is the $n$-th derivative of $f$. Of course, this equality can be extended to functions of two variables as follows;

$$
\begin{gathered}
G_{\alpha}\left(f^{(n)}\right)=\frac{F(x, u)}{u^{n}}-u^{\alpha}\left[\frac{1}{u^{n-1}} f(x, 0)+\frac{1}{u^{n-2}} \frac{\partial}{\partial t} f(x, 0)+\cdots\right. \\
\left.+\frac{1}{u} \frac{\partial^{n-2}}{\partial t^{n-2}} f(x, 0)+\frac{\partial^{n-1}}{\partial t^{n-1}} f(x, 0)\right],
\end{gathered}
$$

where $F(x, u)=G(x, t)$ and $\partial^{0} / \partial=1$. In other hand, the above equality can be represented as theorem 3 .

Theorem 3. If a function $f$ is an integrable, then the transform $f^{(n)}$ satisfies

$$
G_{\alpha}\left[\frac{\partial^{n} f(x, t)}{\partial t^{n}}\right]=\frac{F(x, u)}{u^{n}}-u^{\alpha} \sum_{k=1}^{n} \frac{1}{u^{n-k}} \frac{\partial^{k-1} f(x, 0)}{\partial t^{k-1}},
$$

where $u=G_{\alpha}(f)=F(u)$ and $\partial^{0} / \partial=1[7]$.

Table 1: Table of Laplace-typed integral transform $G$ [4]

\begin{tabular}{ccc}
\hline & $\mathrm{f}(\mathrm{t})$ & $\mathrm{G}(\mathrm{f})$ \\
\hline 1 & 1 & $u^{\alpha+1}$ \\
1 & $\mathrm{t}$ & $u^{\alpha+2}$ \\
3 & $t^{n}$ & $n ! \cdot u^{n+\alpha+1}$ \\
4 & $e^{a t}$ & $\frac{u^{\alpha+1}}{1-a u}$ \\
5 & $\sin a t$ & $\frac{a u^{\alpha+2}}{1+u^{2} a^{2}}$ \\
6 & $\cos a t$ & $\frac{u^{\alpha+1}}{1+u^{2} a^{2}}$ \\
7 & $\sinh a t$ & $\frac{a u^{\alpha+2}}{1-u^{2} a^{2}}$ \\
8 & $\cosh a t$ & $\frac{u^{\alpha+1}}{1-u^{2} a^{2}}$ \\
9 & $e^{a t} \cos b t$ & $\frac{u^{\alpha}\left(\frac{1}{u}-a\right)}{\left(\frac{1}{u}-a\right)^{2}+b^{2}}$ \\
10 & $e^{a t} \sin b t$ & $\frac{b u^{\alpha}}{\left(\frac{1}{u}-a\right)^{2}+b^{2}}$ \\
\hline
\end{tabular}

Conflict of interest The author declare that there is no conflict of interest regarding the publication of this paper.

Acknowledgements This research was supported by Kyungdong University Research Fund, 2020. 


\section{REFERENCES}

[1] Bertrand, J., Bertrand, P., and Ovarlez J.P., The Mellin Transform, The Transforms and Applications, Handbook (A.D. Poularkas, ed.). CRC Press, Boca Raton, FL, 1996.

[2] T. M. Elzaki, S. M. Ezaki and E. M. A. Hilal, ELzaki and Sumudu Transform for Solving some Differential Equations, Glob. J. of Pure \& Appl. Math., 8 (2012), 167-173.

[3] Jhanthanam S, Nonlaopon K, Orankitjaroen S. Generalized Solutions of the Third-Order Cauchy-Euler Equation in the Space of Right-Sided Distributions via Laplace Transform, Mathematics, 7 (2019), 376.

[4] $\mathrm{Hj}$. Kim, The intrinsic structure and properties of Laplace-typed integral transforms, Mathematical Problem in Engineering, 2017 (2017), 1-8.

[5] $\mathrm{Hj}$. Kim, The solution of laguerre's equation by using G-transform, Int. J. of Applied Engineering Research, 24 (2017), 16083-16086.

[6] $\mathrm{Hj}$. Kim, The solution of the heat equation without boundary conditions, Dynamic Systems and Applications, 27 (2018), 653-662.

[7] Hj. Kim, S. Sattaso, K. Kaewnimit, and K. Nonlaopon, An Application of Generalized Laplace Transform in PDEs, Advances in Dynamical Systems and Applications 14 (2019), 257-265

[8] Koepf, Wolfram, Insuk Kim, and Arjun K. Rathie, On a New Class of Laplace-Type Integrals Involving Generalized Hypergeometric Functions, Axioms, 8, (2019), P87.

[9] E. Kreyszig, Advanced Engineering Mathematics, Wiley, Singapore, 2013.

[10] Mohand M. Abdelrahim Mahgoub, The New Integral Transform 'Mohand Transform', Advances in Theoretical and Applied Mathematics 12, (2017), 113-120.

[11] S. Supaknaree, K. Nonlaopon, and Hj. Kim, Further properties of Laplace-type integral transforms, Dynamic Systems and Applications, 28 (2019), 195-215.

[12] G. K. Watugula, Sumudu Transform: a new integral transform to solve differential equations and control engineering problems, Integrated Education, 24 (1993), $35-43$. 